\title{
THE ONE-DIMENSIONAL TURBULENCE ASPECTS OF INTERNAL FORCED CONVECTIVE FLOWS
}

\author{
Juan A. Medina Méndez ${ }^{1 *}$, Marten Klein ${ }^{1}$ and Heiko Schmidt ${ }^{1}$. \\ ${ }^{1}$ Chair of Numerical Fluid and Gas Dynamics, BTU Cottbus-Senftenberg. \\ Anwendungszentrum Fluiddynamik, Siemens-Halske Ring 15a, 03046 Cottbus, Germany. \\ *Contact:medinjua@b-tu.de-https://www.b-tu.de/en/fg-stroemungsmodellierung/
}

Key words: ODT, wall-bounded flows, turbulence modeling, drag, heat transfer

\begin{abstract}
We present an overview of issues for the modeling of internal forced convective flows with the One-Dimensional Turbulence (ODT) model. Results of recent research as well as prospective research issues are presented for statistically streamwise homogeneous flows and streamwise inhomogeneous mixed convective flows. The results illustrate the capabilities of the model to evaluate and bring insight into a wide range of physical phenomena in the field of convective flows. Nonetheless, as a model, ODT is best suited for the evaluation of asymptotically turbulent flows, i.e., away from laminar regimes.
\end{abstract}

\section{INTRODUCTION}

The motivation to study internal flows for turbulence research could be traced all the way back to the experiments of Reynolds. In modern terminology, internal flows can be categorized in several ways. We refer to the categorization in terms of the convective scales. Forced convection is associated to the dominance of motion, generally streamwise motion, caused by an external force. Additionally, we associate forced convection to small convective time scales. This is in comparison to motion induced by the response of the fluid to another transport process, e.g., heat transfer, which would be characteristic of the second group of internal flows in our categorization, natural convective flows.

Understanding of the phenomenology of turbulence in forced convective flows has been greatly aided by Direct Numerical Simulations (DNSs) in the last years. A detailed overview of issues in constant property internal forced convective turbulent flows is presented by Marusic et al. [1]. Many of the issues presented therein refer to the so-called asymptotic wall-bounded turbulent flows, i.e., the limit of turbulent flows at very large Reynolds numbers. An example of the latter, as suggested by [2], would be a channel flow simulation at a friction Reynolds number $R e_{\tau} \sim 10^{4}$. See as a reference the State of the Art channel flow DNS by [3] computed at the expense of petascale computational resources [4]. At low Reynolds numbers, Ávila et al. [5] has brought light into the exact laminar-to-turbulent transitional bulk Reynolds number in pipe flow $R e_{b}=2040\left(R e_{b}\right.$ based on the diameter of the pipe), also aided by State of the Art DNS computations. Thus, very distinctive type of phenomena take place according to the magnitude of $R e$. For relevant issues requiring fundamental understanding, we require extensive computational resources. In a general sense, $R e$ is an important parametrization of turbulence for internal constant property flows. For natural convection flows, investigations have signalized a similar threshold Grashof number for the laminar-to-turbulent transition $G r_{b} \approx 10^{9}$, see [6]. 
It is the objective of this work to propose an alternative low fidelity tool to investigate fundamental issues in wall-bounded turbulent flows. This tool is the One-Dimensional Turbulence model, which was originally formulated by Kerstein in 1999 [7], and has undergone extensive validation in the context of wall-bounded flows so far $[8,9,10,11]$. As a low fidelity turbulence model, which nonetheless has the ability to correctly resolve all time and length scales in a 1-D domain, ODT is an appealing alternative for the support of DNS investigations in parametric spaces, e.g., such as the $R e-G r$ parametric space in convective flows. In this work we will present several issues of the ODT model investigated by the authors, which are all intended to address the problematic of internal forced convective turbulent flows.

\section{OVERVIEW OF THE ODT MODEL}

ODT is a reduced order stochastic turbulence model. Unlike the Probability Density Function (PDF) transport method [12], ODT does not rely on the direct resolution of a Joint PDF (JPDF) transport equation for the velocity and composition of the flow. Nonetheless, ODT does rely on the representation and dynamical changes of a JPDF corresponding to the random process of so-called eddy events in the model. The eddy events are the model representation of turbulent transport. In the case of a constant property solenoidal velocity field exhibiting parabolic features, and one statistically inhomogeneous direction of property gradients setting the reference length and time scales of the turbulent flow, eddy events are the specific representation of turbulent advection. In this context, changes in the flow velocity induced by an ensemble of eddy events can be associated to the Reynolds shear stress [13]. The eddy events in ODT are mapping rules applied on 1-D scalar profiles within a specific range of the computational 1-D domain,
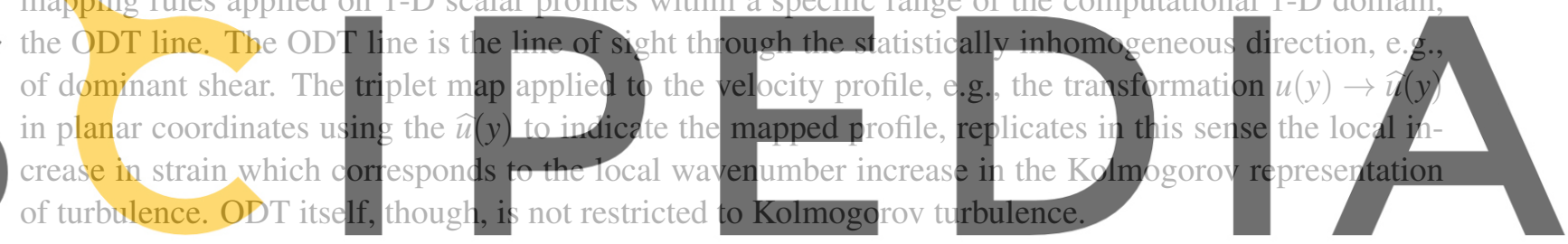

The triplet map applied to scalar profiles in planar Cartesian coordinates has been detailed several times

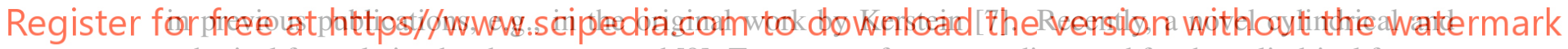
spherical formulation has been proposed [9]. Two types of maps are discussed for the cylindrical formulation, TMA and TMB. In TMA, the triplet map splits the eddy region into thirds by equal cylindrical wedge volumes. The triplet map TMB preserves the similarity with the planar formulation by splitting the eddy region into thirds by equal length. The cylindrical triplet map transformation rule for TMA is detailed in [14]. Stochastic implementation of eddy events requires sampling from a JPDF for the eddy event size $l$, and position $r_{0}$, whereas $h(l)$ and $g\left(r_{0}\right)$ are presumed JPDFs for the sampling procedure. Sampling from presumed distribution functions makes the stochastic process dependent on the form of such distributions. Instead, (a Bernoulli) oversampling from a statistical Poisson process with probability density $\lambda \Delta \xi_{s}$ compared to the presumed probability density $h(l) g\left(r_{0}\right)$ yields a physically convergent process at the limit $\Delta \xi_{s}<\xi$. $\xi$ and $\xi_{s}$ could be either time scales $t_{\text {eddy }}$ and $t_{s}$, or length scales $L_{\text {eddy }}$ and $L_{s}$, noting that $L_{\text {eddy }} \neq l$. These two representations correpond to the temporal T-ODT and spatial S-ODT formulations, respectively [15]. In T-ODT, the ODT line remains at a fixed spatial location and simulates (with sampling rate $\Delta t_{s}$ ) the temporal change of properties from a set of initial conditions within the 1-D domain. In S-ODT, the Lagrangian ODT line advects parabolically in streamwise direction (with sampling rate $\Delta L_{s}$ ) from a set of initial, or entry conditions. We note that $\lambda$ is a dimensionally formulated 
eddy rate of the form,

$$
\lambda=\frac{C}{l^{2} \xi},
$$

where, $C$ is a constant of proportionality defining the dimensional law. This is an ODT model parameter, generally associated to the turbulence intensity, i.e., to the frequency of eddy events. Another model parameter of interest, $Z$, is found on the model equation for the calculation of $\xi$; specializing for the T-ODT formulation, this reads [14],

$$
\frac{1}{2 K_{0}}\left(\frac{1}{t_{\text {eddy }}}\right)^{2} \int_{l} \hat{\rho} K^{2} \mathrm{~d} \forall=K_{0}\left(\sum_{k} \Xi_{\mathrm{kin}, \text { eddy }, k}^{\prime \prime}\right)-Z \Xi_{\mathrm{vp}, \text { eddy }} .
$$

Here, $K$ is the ODT kernel function defined as $K=\chi-f(\chi)$, where $\chi$ is a placeholder indicating the coordinate of the ODT line, e.g., $\chi=y$ in planar Cartesian coordinates and $\chi=r$ for cylindrical coordinates. Similarly, the effective volume differential dV is a placeholder for the measure of the differential elements in the transformation. This is a linear measure of length in planar coordinates $\mathrm{dV}=\mathrm{d} y$ for ODT lines coinciding with the $y$ direction, and a nonlinear, radially weighted length measure in cylindrical coordinates $\mathrm{d} \forall=r \mathrm{~d} r$, for radially oriented ODT lines. The terms $\Xi_{\text {kin,eddy }, k}$ and $\Xi_{\mathrm{vp}, \text { eddy }}$ are model representations of the available kinetic energy for the eddy event according to the velocity profiles, and the kinetic energy dissipation as heat from the smallest representable eddies, respectively. In this context, the subindex $k$ corresponds to the velocity component $u_{k}$ of the vector velocity field $V=[u, v, w]^{T}$.

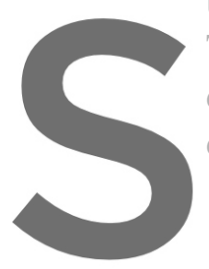
Therefore, $Z$ is a proportionality dissipation. Together w displacement, the mode
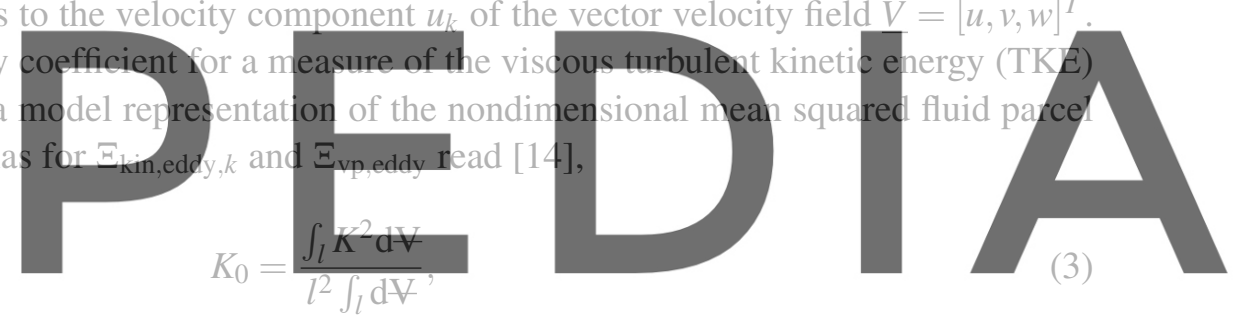

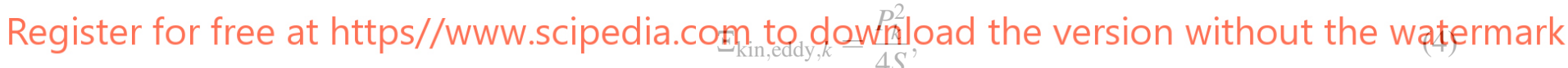

$$
\Xi_{\mathrm{vp}, \text { eddy }}=\frac{\mu_{\text {eddy }}^{2} \int_{l} \mathrm{dV}}{2 \rho_{\text {eddy }} l^{2}},
$$

where the quantities $P_{k}$ and $S$ have been defined as,

$$
\begin{aligned}
P_{k} & =\int_{l} \widehat{\rho} \widehat{u_{k}} K \mathrm{~d} \mathrm{~V}-\frac{\rho_{K}}{\rho_{J}} \int_{l} \widehat{\rho} \widehat{u_{k}} J \mathrm{~d} \mathrm{~V}, \\
S & =\frac{1}{2}\left(\frac{\rho_{K}^{2}}{\rho_{J}^{2}}+1\right) \rho_{K K}-\frac{\rho_{K}}{\rho_{J}} \rho_{J K} .
\end{aligned}
$$

In the previous equations, $J$ is a second kernel function $J=|K|$, and $\rho_{K}, \rho_{J}, \rho_{J K}$ and $\rho_{K K}$ are kernel weighted integrals of the density, i.e., $\int_{l} \widehat{\rho} K \mathrm{dV}, \int_{l} \widehat{\rho} J \mathrm{dV}, \int_{l} \widehat{\rho} J K \mathrm{~d} V$, and $\int_{l} \widehat{\rho} K^{2} \mathrm{dV}$, respectively. Finally, $\mu_{\text {eddy }}$ and $\rho_{\text {eddy }}$ are filtered values of the dynamic viscosity and the density within the eddy event range $l$.

For a better physical representation of the effects of the turbulent transport in the ODT formulation with velocity components $u_{k}$, a kinetic energy redistribution mechanism takes place after the mapping 
of velocity components, see [13]. That is, each velocity component is mapped and modified according to the kernel functions $J$ and $K$ weighted by uniform coefficients, i.e., the final velocity component after an eddy event is $\widehat{u_{k}}=b_{k} J+c_{k} K$. This yields the third model parameter in ODT, $\alpha$, with $\alpha \in[0,1]$, associated to the energy redistribution mechanism. Details of the calculation formulas for the generalized S-ODT and T-ODT formulations can be found in [14]. Specializing again to the T-ODT formulation, $b_{k}=-c_{k}\left(\rho_{K} / \rho_{J}\right)$. Using $m, n$ and $o$ as a permutation of the indices 1,2 and 3 , and $\operatorname{sgn}()$ as the sign operator, $c_{m}$ is calculated as

$$
c_{m}=\frac{1}{2 S}\left[-P_{m}+\operatorname{sgn}\left(P_{m}\right) \sqrt{(1-\alpha) P_{m}^{2}+\frac{\alpha}{2}\left(P_{n}^{2}+P_{o}^{2}\right)}\right] .
$$

A continuous evolution of the JPDF of eddy events in time in T-ODT (or space in S-ODT) takes place by advancing deterministic 1-D Partial Differential Equations (PDEs) for the velocity components in time (or streamwise direction), after the successful implementation of an eddy event. The 1-D PDEs also allow the incorporation of the convective and diffusive transport; the latter one associated to the viscous effects on the flow momentum. The PDE governing the material evolution of fluid parcel momentum between eddy events, specializing to planar coordinates, can be written as,

$$
\rho \frac{\mathrm{D} u_{k}}{\mathrm{D} t}=-\frac{\mathrm{d} \bar{p}}{\mathrm{~d} x} \delta_{1 k}+\frac{\partial}{\partial y}\left(\mu \frac{\partial u_{k}}{\partial y}\right)
$$

Here, $\rho$ and $\mu$ are the local density and dynamic viscosity, while $\mathrm{d} \bar{p} / \mathrm{d} x$ is a uniform momentum source for the streamwise velocity component. This is implemented either by suggesting a constant value, as
in Fixed Pressure Gradient (FPG) driven internal flows, or by enforcing a corretion in the velocity ffeld
in order to comply with a constant bulk avernged mass flux in the 1-D domain during the determifistic
advancement, as in Constant Flow Rate (CFR) driven internal flows. The material derivative D/Dt is
interpreted as a temporal rate of change d/dt in the T-ODT formulation, and as streamwise convertive derivative $u(\mathrm{~d} / \mathrm{d} x)$ in the S-ODT formulation. This completes the description of the generalized vector

\section{Register for free at https//WWW.scipedia.com to download the version without the watermark}

\section{STATISTICALLY STATIONARY AND STREAMWISE HOMOGENEOUS FLOWS}

To illustrate the model capabilities, we first refer to statistically stationary and streamwise homogeneous internal flows. This is the classical case of fully developed turbulent pipe or channel flows. As a validation case, we evaluated three different friction Reynolds numbers with both T-ODT and S-ODT formulations. For a statistically stationary, streamwise and spanwise homogeneous flow such as a channel flow, or a statistically stationary, streamwise and circumferential homogeneous flow such as a pipe flow, temporal statistics are equal to streamwise statistics. Thus, we can evaluate the performance of both T-ODT and S-ODT formulations against DNS data in such cases. $R e_{\tau}=\rho u_{\tau} \delta / \mu$ is based on the friction velocity $u_{\tau}$ and the thickness $\delta$, where $\delta$ is the channel half-height, or the radius in a pipe. The ODT model parameters for the T-ODT channel flow simulations are $C=6.5, Z=300$ and $\alpha=2 / 3$, while for pipe flow simulations $C=5, Z=350$ and $\alpha=0$. Further inputs for the model, concerning restrictions on the maximum allowed eddy sizes, and adaptive mesh control parameters [8] are detailed in [14]. For S-ODT simulations, both pipe and channel flow simulations use $C=3, Z=100$ and $\alpha=0$. We note that the S-ODT formulation used here corresponds to the non conservative formulation detailed in $[11,14]$. 

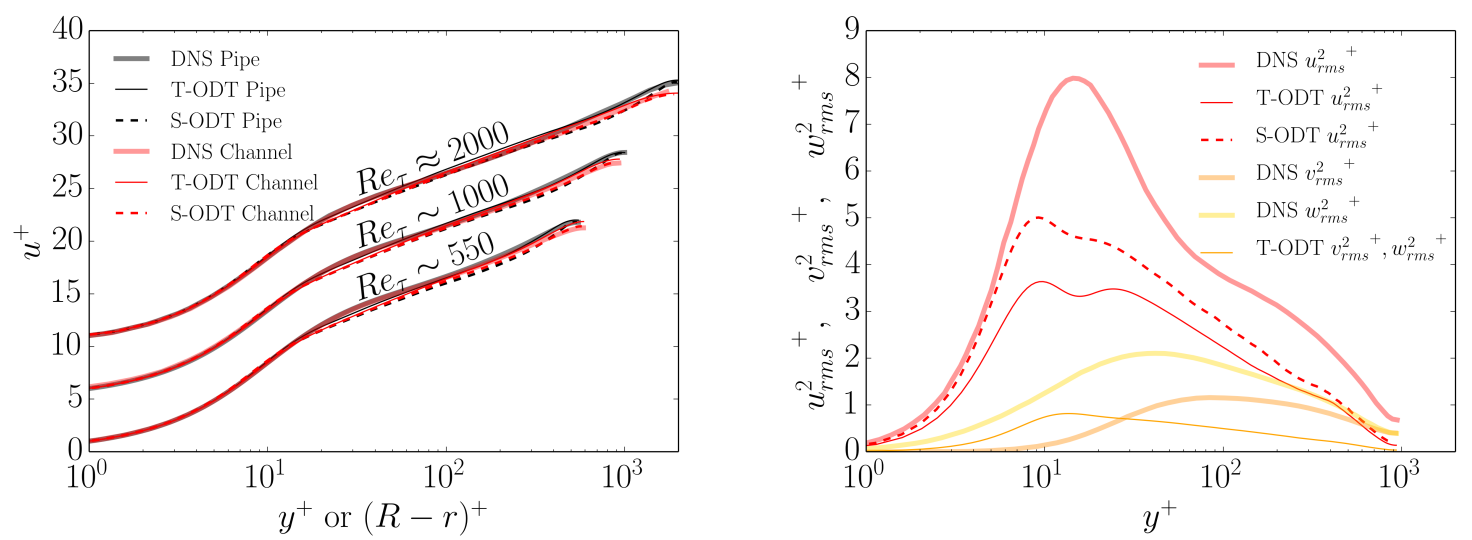

Figure 1: [left] Mean streamwise velocity profiles in wall-normal units at channel flow friction Reynolds numbers $R e_{\tau}=590, R e_{\tau}=934$ and $R e_{\tau}=2000$, and pipe flow Reynolds numbers $R e_{\tau}=550, R e_{\tau}=1000$ and $R e_{\tau}=2000$. The profiles at larger Reynolds numbers are shifted upwards by $u^{+}=5$ for better visualization. [right] Squared RMS velocity profiles in wall-normal units for channel flow simulations at $R e_{\tau}=934$. DNS reference data from [16] (channel flow $R e_{\tau}=550$ ), [17] (pipe flow $\left.R e_{\tau}=550, R e_{\tau}=1000\right)$, [18] (channel flow $R e_{\tau}=934, R e_{\tau}=2000$ ), and [19] (pipe flow $R e_{\tau}=2000$ ) is shown for reference.

This is explained in section 5. Nonetheless, the selection $\alpha=0$ is intentional in this sense, given that it is the only choice for which the formulation from $[11,14]$ becomes conservative in constant property

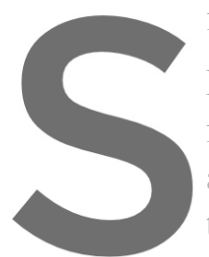
Figure 1 [left] shows the mean s friction Reynolds number available reference DNS the T-ODT and S-ODT and streamwise homogeneous flows, both in planar and cylindrical coor
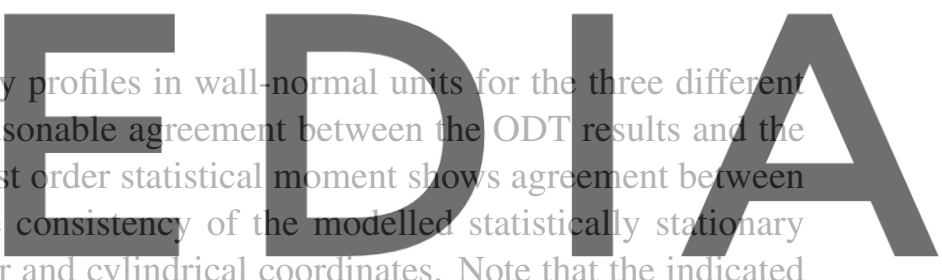

shows the RMS velocity profiles for the channel flow configuration at $R e_{\tau} \sim 1000$. It is noted that the nondimensional $u_{r m s}$ profile is the only one shown for the S-ODT formulation. This is due to the choose $\alpha=0$ and the assumption of zero uniform initial conditions for the velocity components $v$ and $w$, such that no energy redistribution takes place between $u$ and the components $v$ and $w$. Similarly, the choice $\alpha=2 / 3$ and the use of similar zero uniform initial conditions for $v$ and $w$ is the reason why the velocity profiles $v_{r m s}$ and $w_{r m s}$ perfectly overlap in the T-ODT formulation. That is, the kinetic energy from $u$ is equally redistributed to $v$ and $w$ during eddy events by the kernel mechanism. Both T-ODT and SODT formulations underestimate the RMS velocity profiles, although the S-ODT formulation produces enhanced levels of turbulence intensity, and does not feature the double peak close to the wall for $u_{r m s}$ observed in the T-ODT formulation [8].

\section{INTERNAL FLOWS WITH HEAT TRANSFER}

In contrast to the constant property forced convection simulations reported in section 3 , we now report on T-ODT and S-ODT pipe flow simulations with a non negligible heat transfer. These simulations 
correspond to mixed convection simulations of an upward pipe flow of air (as an ideal gas) with a fixed nondimensional heat flux $q^{*}=q_{w} /\left(\rho_{0} U_{b, 0} T_{0} c_{p, 0}\right)=0.0018$, where $q_{w}$ is the (dimensionally consistent) heat flux at the wall, $T_{0}, \rho_{0}, c_{p, 0}$ and $U_{b, 0}$ are the initial uniform temperature and corresponding initial density, specific heat capacity at constant pressure, and initial bulk velocity. Initial conditions for the pipe flow velocity field are fully developed T-ODT constant property pipe flow simulations for a target initial bulk Reynolds number $R e_{b, 0}=\rho_{0} U_{b, 0} D / \mu_{0} \approx 6000$, where $\mu_{0}$ is the initial dynamic viscosity and $D$ the diameter of the pipe $(D=0.0274 \mathrm{~m}) . T_{0}=298.15 \mathrm{~K}$ and the uniform (and constant) thermodynamic pressure is set to $P_{0}=100 \mathrm{kPa}$. The ODT model parameters are taken as $C=3.5, Z=350, \alpha=2 / 3$ for the T-ODT simulations, and $C=2.5, Z=100, \alpha=2 / 3$ for the S-ODT simulations. Further details for the simulation inputs can be found in [11,14]. As in section 3, the S-ODT formulation used in this case is inherently non conservative. Heat transfer effects are incorporated into the formulation by the solution of a temperature PDE, as well as a modified divergence condition for the velocity field. The latter divergence condition reduces to a gradient condition on a dilatational velocity, which dictates cell compression and expansion in the 1-D domain as a consequence of the temperature and density changes. Following $[11,14]$, equation 9 in the S-ODT interpretation considers D/Dt as an advective derivative $u_{D}(\mathrm{~d} / \mathrm{d} x)$, where $u_{D}$ is a numerically delayed streamwise velocity, which is interpreted as part of the dilatational velocity field. In that sense, the gradient condition for the dilatational velocity component in the ODT line direction $v_{D}$, and the temperature PDE required in the heat transfer cases are written for cylindrical coordinates, as in [11],
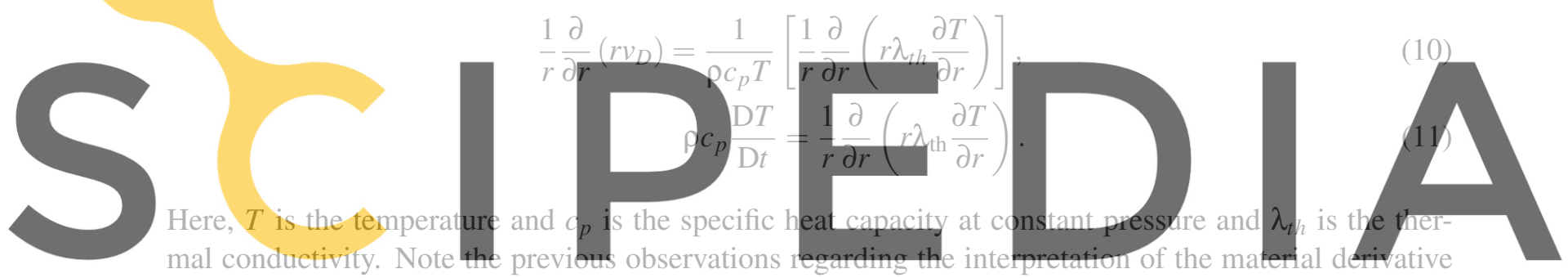

notation as in Eq. 9. Figure 2 [left] shows the T-ODT generated temperature field. Note that the field is

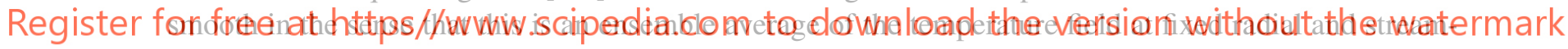
wise positions, with a number of ensemble members $N_{\text {ens }}=4800$. Also, the T-ODT formulation is not able to obtain streamwise solutions directly. This is done instead by means of the Taylor's hypothesis [20], displacing the temporal solutions in the streamwise vertical coordinate $z$ with the help of the bulk velocity $U_{b}$, where $U_{b}$ is obtained from the CFR condition and the correspondent bulk density [14],

$$
z\left(t_{0}+\Delta t\right)=z\left(t_{0}\right)+\int_{t_{0}}^{t_{0}+\Delta t} U_{b}\left(t^{\prime}\right) \mathrm{d} t^{\prime}
$$

Figure 2 [right] shows the S-ODT (ensemble averaged) generated temperature field. In comparison to T-ODT, the temperature gradient at the wall is more pronounced in S-ODT, and the overall outer layer of the flow is not as diffused and uniform as in T-ODT. The larger temperature gradient in S-ODT allows a more faithful representation of the wall temperature, and of the heat transport characterization by convection and conduction, i.e., the Nusselt number, see [11, 14]. The better reproducibility of the temperature gradient is also the reason why the S-ODT formulation is able, in general, to provide more accurate temperature profiles than the T-ODT counterpart in comparison to DNS data. This is seen in Figure 3 [left]. It is noted, however, that the representation of velocity profiles is not significatively 

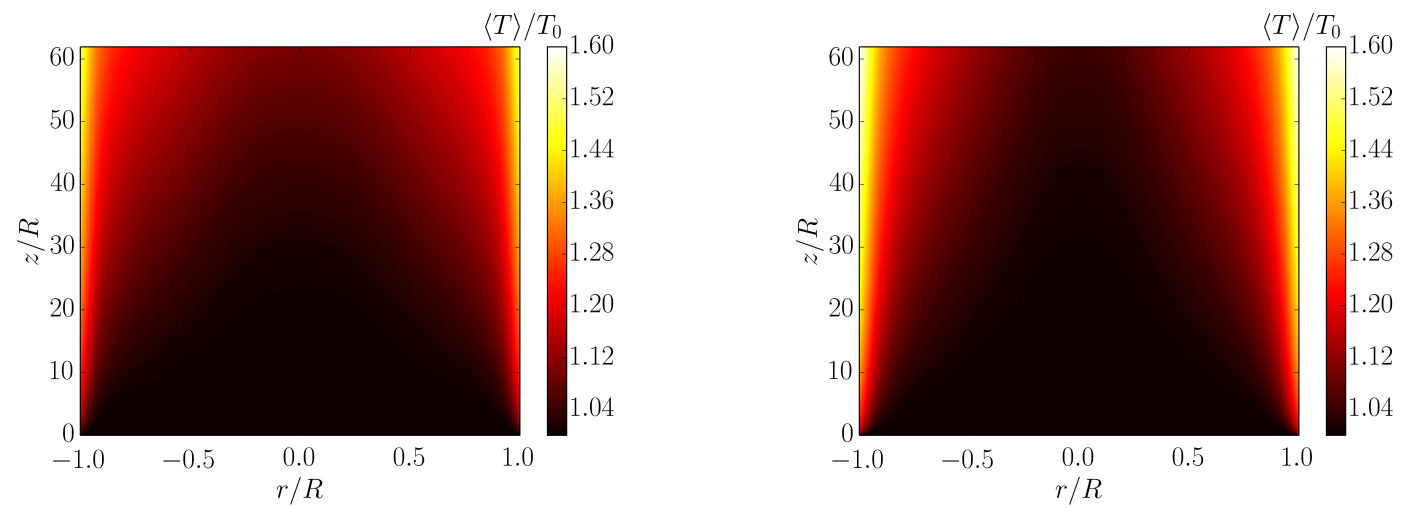

Figure 2: Normalized coordinate ensemble averaged temperature field $\langle T\rangle$ in the upward heated pipe flow. Spatial coordinates have been normalized by the radius of the pipe $R$, while the temperature field has been normalized by the initial uniform temperature $T_{0}$. The [left] Figure shows the temperature field obtained with the T-ODT formulation, and the [right] figure shows the S-ODT temperature field.

different, e.g., see the Van Driest velocity profiles in Figure 3 [right]. That is, although the temperature itself can not be aecurately captured by the use of the Taylor's hypothesis in the T-ODT formulation due to the very large temperature gradients [21], the modification of fluid properties due to temperature still affects in a negligible way the transport of momentum.
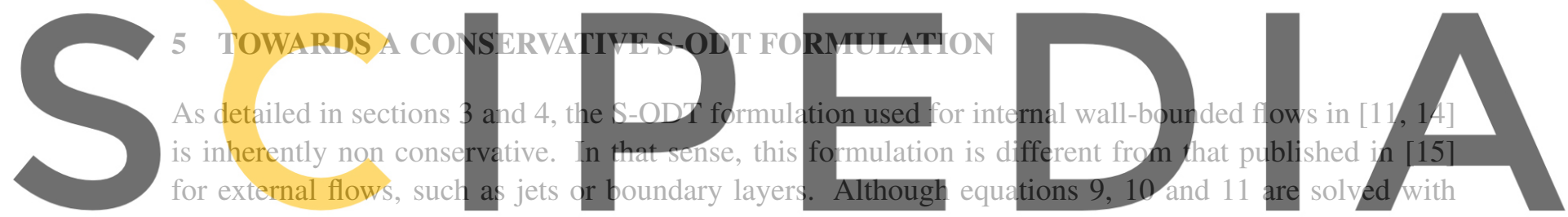

a Finite Volume Method (FVM), resolved integrals for momentum and energy do not coincide with the

Register for frence velacity fyeld in equation 1 thus resulting in the mentioned conservation issues This is most equation and the ideal gas law for a 2-D dilatational vector velocity field $V_{D}=\left[u_{D}, v_{D}, 0\right]^{T}$

$$
\nabla \cdot \underline{V}_{D}=-\frac{1}{\rho} \frac{\mathrm{D} \rho}{\mathrm{D} t}=\frac{1}{\rho c_{p} T}\left(\rho c_{p} \frac{\mathrm{D} T}{\mathrm{D} t}\right) .
$$

Here, the first equality corresponds to the generalized differential law for conservation of mass, while the second equality results from the application of the ideal gas law at fixed gas composition and thermodynamic pressure. Attempting to integrate within a finite volume the LHS and RHS of equation 13 results in the fact that the integration $\int\left(\nabla \cdot \underline{V}_{D}\right) \mathrm{d} V$ corresponds with the integration of $\int\left(\rho c_{p} \mathrm{D} T / \mathrm{D} t\right) \mathrm{dV}$, only for the case that $c_{p}$ is a (uniform) constant, given that $\rho c_{p} T=P_{0} c_{p} / R_{\mathrm{gas}}$ by the ideal gas law, where $R_{\mathrm{gas}}$ is the specific gas constant. Note that this failure to conserve integral properties is not exclusive to the variable density S-ODT formulation, but rather, to any formulation resorting to the use of the velocity divergence condition, e.g., the T-DOT formulation in [23]. Figure 4 [left] shows the spatial variation of $c_{p}$ for two different normalized streamwise positions, $z / R \sim 2$ and $z / R \sim 60$, which are close to the beginning and end of the S-ODT simulations shown in section 4 . Despite the inhomogeneity of $c_{p}$ in the 1-D domain, the maximum norm for the deviation with respect to the bulk uniform $c_{p, b}$ is $2 \%$ in the 

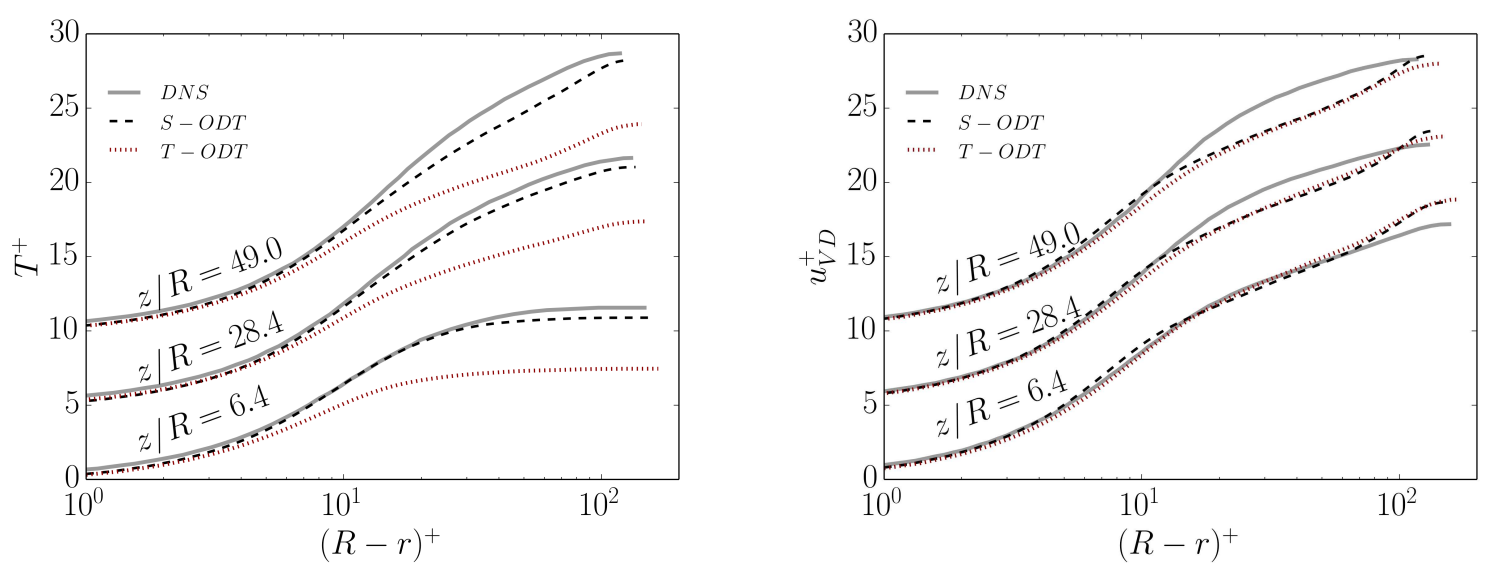

Figure 3: [left] Mean temperature profiles in wall-normal units at different normalized streamwise positions. Note the normalization $T^{+}=\left(\left\langle T_{w}\right\rangle-\langle T\rangle\right) / T_{\tau}$, where $T_{\tau}=q_{w} /\left(\left\langle\rho_{w}\right\rangle\left\langle c_{p, w}\right\rangle u_{\tau}\right)$ is the friction temperature. $\left\langle T_{w}\right\rangle,\left\langle\rho_{w}\right\rangle$ and $\left\langle c_{p, w}\right\rangle$ are the ensemble averaged temperature, density and specific heat capacity at the wall. Wall-normal coordinates are normalized by the traditional viscous length scale. [right] Van Driest velocity profiles in wallnormal units at different normalized streamwise positions. Note that $u_{V D}^{+}=u_{V D} / u_{\tau}$, where the Van Driest velocity is calculated as $u_{V D}=\int_{\psi=0}^{\psi=\langle u\rangle}\left(\langle\rho\rangle /\left\langle\rho_{w}\right\rangle\right) \mathrm{d} \psi$. Profiles at higher streamwise coordinates $z / R$ are shifted upwards by $T^{+}=5$ or $u_{V D}^{+}=5$ for better visualization. DNS data from [22] is shown for reference.

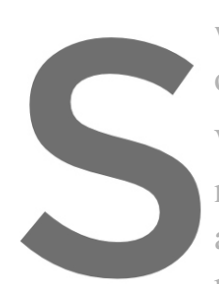

worst case scenario, which

obtained in [11].

We return now to the constant property S-ODT sinn

now with the choice $\alpha$

and hence, conservation

reflects the changes induced by eddy events in S-
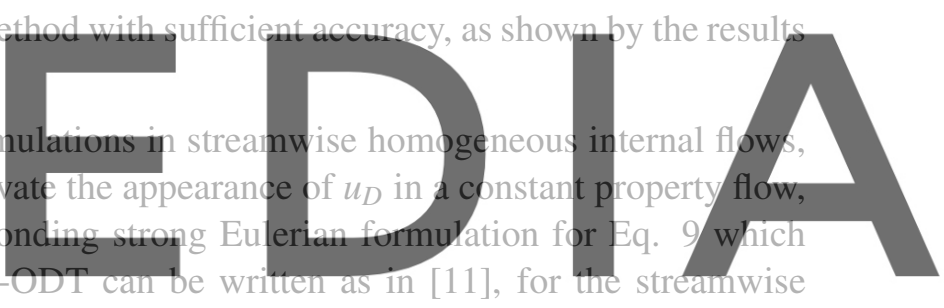

velocity component $u$ in cylindrical coordinates as,

Register for free at https//www_scipedia.com to download the version without the watermark $\frac{\partial\left(u_{D} u\right)}{\partial z}=-\frac{\partial p}{\partial z}+\frac{1}{r}\left(r \mu \frac{\partial u}{\partial r}\right)+M_{u}+T_{u}$.

$M_{u}+T_{u}$ corresponds to the modeled effects of turbulent advection by triplet maps and kernel redistribution mechanisms on $u$ [13]. In a conservative formulation, by averaging equation $14, \overline{M_{u}}+\overline{T_{u}}$ should equate the Reynolds stress $\overline{u^{\prime} v^{\prime}}$, i.e.,

$$
\overline{u^{\prime} v^{\prime}}=\frac{1}{r} \int \frac{1}{\rho}\left(\overline{M_{u}}+\overline{T_{u}}\right) r \mathrm{~d} r .
$$

On the absence of deterministic advancement, this implies that $\overline{M_{u}}+\overline{T_{u}}$ is evaluated as the cumulative eddy induced changes on $\Delta\left(\rho u_{D} u\right)$ over an interval $\Delta z$, as per equation 14 . This estimation of the Reynolds stress should coincide with the integration of the Reynolds-averaged Navier-Stokes (RANS) streamwise momentum equation, e.g., for an integration range of the computational domain with left integration limit $-R$ and open variable integration limit $r$, considering $\tau_{w}$ as the wall shear stress and the kinematic viscosity $v=\mu / \rho$,

$$
\overline{u^{\prime} v^{\prime}}=\frac{\tau_{w}}{\rho R} r+v \frac{\partial u}{\partial r}-\frac{\tau_{w}}{\rho}
$$



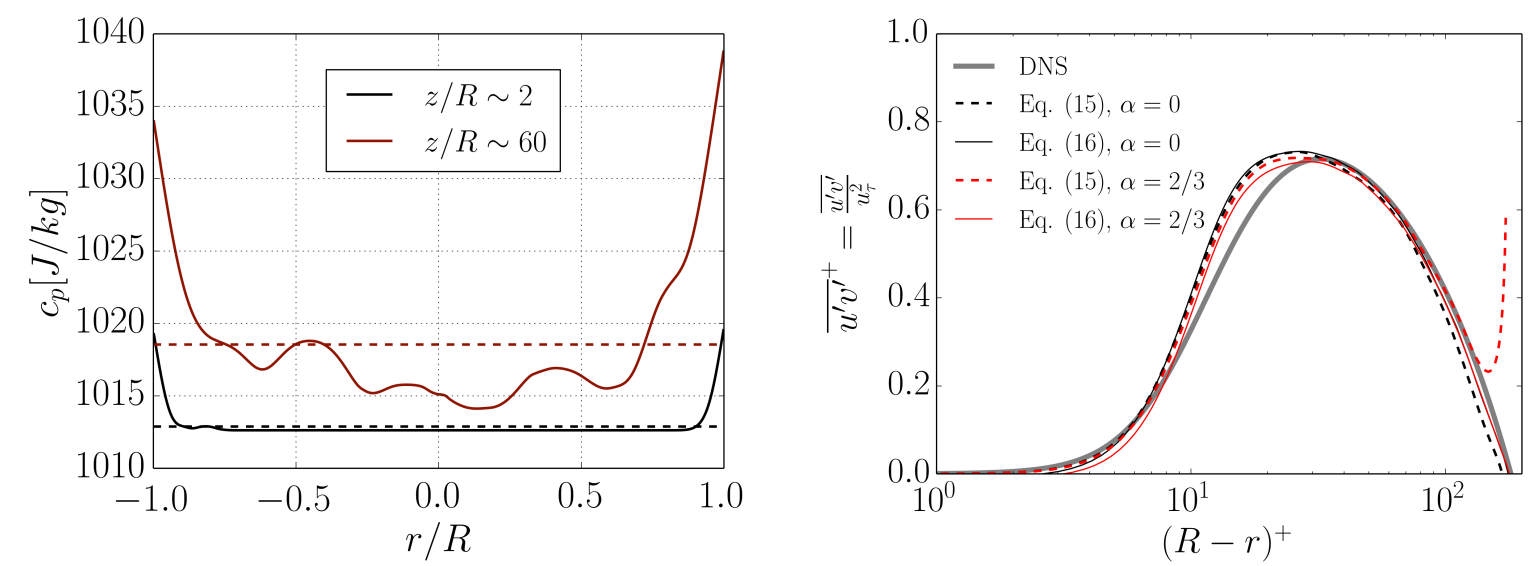

Figure 4: [left] Local radial profiles of $c_{p}$ for one ensemble member of the S-ODT simulations in section 4 at two different streamwise positions $z / R \sim 2$ and $z / R \sim 60$. The thick solid line indicates the profile and the dashed line indicates the bulk average $c_{p, b}$. [right] Reynolds stress calculation methods in a constant property pipe flow S-ODT simulation at $R e_{\tau}=180$. DNS data from [17] is shown for reference.

Figure 4 [right] shows the obtained Reynolds stress for a constant property pipe flow S-ODT simulation at $R e_{\tau}=180$, using the model parameters $C=2.5$ and $Z=100$. The calculated Reynolds stress was obtained assuming $u_{D}=u$, in order to verify the conservation issues. In the conservative case, $\alpha=0$, the calculation of the Reynolds stress by equation 15 mostly coincides with the integration method of equation 17. Differences are attributed to the mesh adaption dynamies in the solver [8]. In the non
conservative case with $\alpha \neq 0$, the calculation methods differ and a non-zero Reynolds stress is obtained
close to $r=0$. A conservative S-ODT formulation for internal forced convective flows should, therefore,
avoid the use of the decomposition of the velocity field into dilatational and solenoidal components, and
also, avoid the use of a velocity divergence condition. Instead, the integral form of continuity should be used as in the S-ODT formulation from [15]. In the Lagrangian representation, this is, for conservation

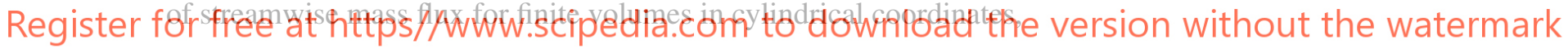

$$
\frac{\mathrm{d}}{\mathrm{d} x} \int \rho \operatorname{pur} \mathrm{d} r=0 \text {. }
$$

Although a first order approximation such as that performed for external flows results in an expanded or contracted domain, a numerical corrector step can be enforced afterwards to comply with the volume constraint, by rescaling the velocity profile $u$ with a uniform scalar factor $\Gamma$. This is work in progress that will be published elsewhere in the future.

\section{ON THE DETERMINATION OF THE ODT MODEL PARAMETERS}

ODT model parameters $C$ and $Z$ used in all simulations presented here so far, as well as in all ODT publications up to date, were obtained following a trial and error procedure, in order to match specific statistical moments of the flow, generally with respect to DNS or experimental data. The model parameter $\alpha$ in this work, is not subject to such calibration procedure, since only two theoretically justifiable values of $\alpha$ are considered. $\alpha=0$ corresponds to zero energy redistribution between velocity components, i.e., a purely scalar one-dimensional turbulence, and $\alpha=2 / 3$ corresponds to equipartition of the available 
kinetic energy between velocity components after an eddy event in order to induce a return to isotropy.

As in RANS or Large Eddy Simulation (LES) models, physically motivated ODT model parameters could facilitate the understanding of the model, and future model extensions for more complex physics. In fact, it could be possible, in theory, to motivate a derivation of the ODT model parameters based on the same reasoning used for some RANS or LES models. Consider, as an example, the universal scaling governing constant property and streamwise homogeneous wall-bounded flows, as presented by [24],

$$
\bar{u}=u_{\tau} F_{0}\left(\frac{y}{\delta}, R e_{\tau}\right) .
$$

Here, $F_{0}$ is a universal dimensionless function. In fact, choosing the correspondence $\bar{u}=U_{b}$ or $\bar{u}=U_{\mathrm{cl}}$, the bulk velocity or the centerline velocity scale, leads to the definition of $F_{0}$ either as $C_{\mathrm{f}}$ or $c_{\mathrm{f}}$, the skin friction coefficient based on $U_{b}$ or $U_{\mathrm{cl}}$. Therefore, a model parameter such as $C$ should be a function of the skin friction coefficient.

As a further example for the estimation of $Z$, we refer to the reduced version of equation 2 for constant density flows, with the definition $u_{k, K}=\left[1 /\left(l \int_{l} \mathrm{dV}\right)\right] \int_{l} \widehat{u_{k}} K \mathrm{dV}$,

$$
\frac{1}{t_{\text {eddy }}}=\frac{v}{l^{2}} \sqrt{\sum_{k=1}^{3}\left(\frac{u_{k, K} l}{v}\right)^{2}-Z}
$$

Choosing $Z=\sum_{k=1}^{3}\left(u_{k, K} l / v\right)^{2}$ sets the square root to zero and prevents implementation of eddy events, i.e., laminarizes the flow. Specifically for the case of channel flow, as an example, choosing an eddy

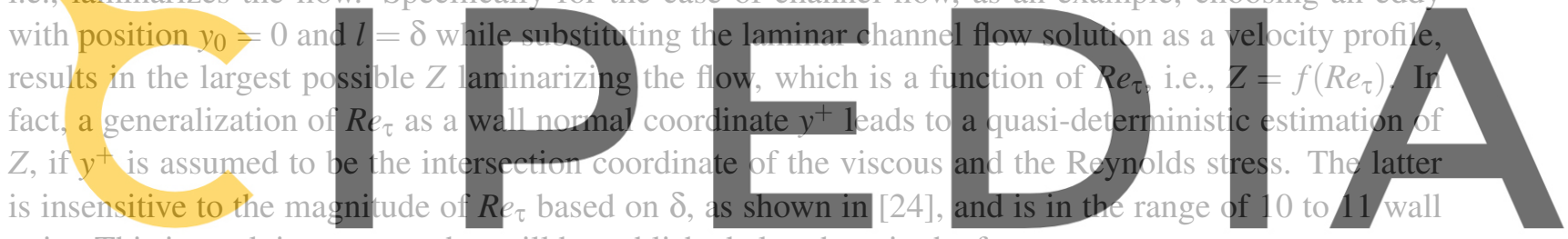
units. This is work in progress that will be published elsewhere in the future.

\section{Register for free at https//www.scipedia.com to download the version without the watermark 7 CLOSING COMMENTS}

We have presented an overview of issues for modeling of internal forced convective flows with ODT. In general, ODT is best suited for the evaluation of asymptotic turbulent flows. For asymptotic wallbounded turbulence [1], this corresponds to turbulent flows defined solely on the base of $R e_{\tau}$. However, asymptotically turbulent flows defined solely on the base of $G r_{b}$, as in natural convective flows, could also be evaluated with ODT. This could clarify relevant State of the Art questions, e.g., the existence of the so-called ultimate regime of thermal convection [25]. ODT simulations such as those presented in section 4 are challenging, given that they are not asymptotically turbulent, they fall within the mixed convection zone such that a $R e_{\tau}$ and $G r_{b}$ dependency arises, and they are close to an alleged relaminarization boundary, see the DNS discussion in [22]. S-ODT is, in that sense, an advantageous formulation which needs to undergo further research. See also Figure 5 for a sketch concerning ideally applicable regimes for ODT. Overall, a low fidelity model such as ODT is very appealing due to its computational efficiency in the treatment of physical flow phenomena with a reasonable degree of accuracy, as we have shown here. Furthermore, the simplified physical treatment in ODT also allows a better understanding of fundamental issues of the turbulence phenomenology. 


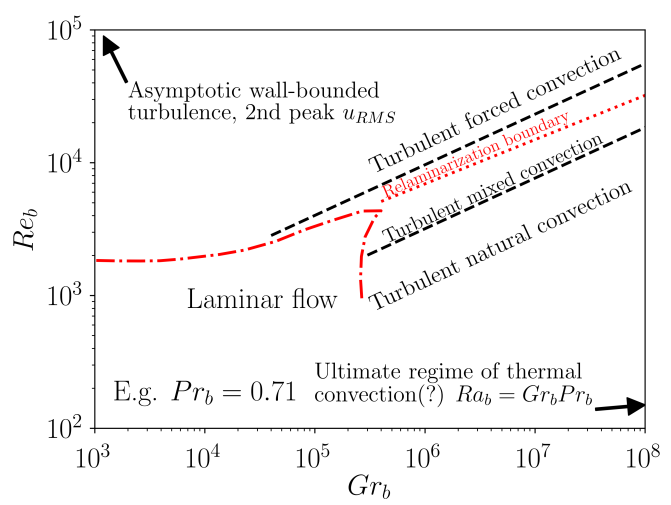

Figure 5: Regime diagram for convective flows. The regime boundaries are sketched following [26]. $P r_{b}$ and $R a_{b}$ correspond to bulk Prandtl and Rayleigh numbers, respectively. The sketch is shown for air, $\operatorname{Pr}_{b}=0.71$.

\section{Acknowledgements}

This work was supported by the European Regional Development Fund (EFRE), grant no. STaF 23035000.

\section{REFERENCES}

[1] I. Marusic, B. J. McKeon, P. A. Monkewitz, H. M. Nagib, A. J. Smits and K. R. Sreenivasan. Wallbounded turbulent flows at high Reynolds numbers: recent advances and key issues. Phys. Fluids (2010) 22(6):065103.

[2] J. Jiménez. Computing high-Reynolds-number turbulence: will simulations ever replace experiments?. J. Turbul. (2003) 4:N22.

[3] M. Lee and R. Moser. Direct Numerical Simulation of turbulent channel flow up to $R e_{\tau}=5200 . J$. Fluid Mech. (2015) 774:395-415.

[4] M. Lee, N. Malaya and R. D. Moser. Petascale direct numerical simulation of turbulent channel flow on up to 786K cores. SC '13: Proceedings of the International Conference on High Performance Computing, Networking, Storage and Analysis (2013).

[5] K. Avila, D. Moxey, A. Lozar, M. Ávila, D. Barkley and B. Hof. The Onset of Turbulence in Pipe Flow. Science (2011) 333:192-196.

[6] G.-U. Kang and B.-J. Chung. The experimental study on transition criteria of natural convection inside a vertical pipe. Int. Commun. Heat Mass Transfer (2010) 37(8):1057-1063.

[7] A. R. Kerstein. One-dimensional turbulence: model formulation and application to homogeneous turbulence, shear flows, and buoyant stratified flows. J. Fluid Mech. (1999) 392:277-334.

[8] D. Lignell, A. Kerstein, G. Sun and E. Monson. Mesh adaption for efficient multiscale implementation of One-Dimensional Turbulence. Theor. Comput. Fluid Dyn. (2013) 27(3-4):273-295.

[9] D. Lignell, V. B. Lansinger, J. A. Medina Méndez, M. Klein, A. R. Kerstein, H. Schmidt, M. Fistler and M. Oevermann. One-dimensional turbulence modeling for cylindrical and spherical flows: model formulation and application. Theor. Comput. Fluid Dyn. (2018) 32(4):495-520. 
[10] Rakhi, M. Klein, J. A. Medina Méndez and H. Schmidt. One-dimensional turbulence modelling of incompressible temporally developing turbulent boundary layers with comparison to DNS. $J$. Turbul. (2019) 20(8):506-543.

[11] J. A. Medina Méndez, M. Klein and H. Schmidt. One-Dimensional Turbulence investigation of variable density effects due to heat transfer in a low Mach number internal air flow. Int. J. Heat Fluid Flow (2019) 80:108481.

[12] S. B. Pope. PDF methods for turbulent reactive flows. Prog. Energy Combust. Sci. (1985) 11(2):119192.

[13] A. R. Kerstein, W. T. Ashurst, S. Wunsch and V. Nilsen. One-dimensional turbulence: Vector formulation and application to free-shear flows. J. Fluid Mech. (2001) 447:85-109.

[14] Medina Mendez, J. A. Application of the One-Dimensional Turbulence model to electrohydrodynamically enhanced internally forced convective flows. $\mathrm{PhD}$ Thesis. Brandenburg University of Technology Cottbus-Senftenberg (2020).

[15] W. T. Ashurst and A. R. Kerstein. One-dimensional turbulence: Variable-density formulation and application to mixing layers. Phys. Fluids (2005) 17(2):025107.

[16] R. Moser, J. Kim and N. Mansour. Direct Numerical Simulation of turbulent channel flow up to $R e_{\tau}=590$. Phys. Fluids (1999) 11(4):943-945.

[17] G. K. El Khoury, P. Schlatter, A. Noorani, P. F. Fischer, G. Brethouwer and A. V. Johansson. Direct Numerical Simulation of Turbulent Pipe Flow at Moderately High Reynolds Numbers. Flow, Turbul. Comb. (2013) 91(3):475-495.

[18] S. Hoyas and J. Jiménez. Scaling of the velocity fluctuations in turbulent channels up to $R e_{\tau}=2003$. Phys. Fluids (2006) 18:011702.

[19] C. Chin, J. P. Monty and A. Ooi. Reynolds number effects in DNS of pipe flow and comparison with channels and boundary layers. Int. J. Heat Fluid Flow (2014) 45:33-40.

[20] C. Geng, G. He, Y. Wang, C. Xu, A. Lozano-Durán and J. M. Wallace. Taylor's hypothesis in turbulent channel flow considered using a transport equation analysis. Phys. Fluids (2015) 27(2):025111.

[21] J. L. Lumley. Interpretation of Time Spectra Measured in High-Intensity Shear Flows. Phys. Fluids (1965) 8(6):1056-1062.

[22] J. H. Bae, J. Y. Yoo, H. Choi and D. M. McEligot. Effects of large density variation on strongly heated internal air flows. Phys. Fluids (2006) 18(7):075102.

[23] J. A. Medina Méndez, H. Schmidt, F. Mauss and Z. Jozefik. Constant volume n-Heptane autoignition using One-Dimensional Turbulence. Combust. Flame (2018) 190:388-401.

[24] S. B. Pope. Turbulent flows. IOP Publishing (2001).

[25] M. Klein and H. Schmidt. Investigating Rayleigh-Bénard convection at low Prandtl numbers using One-Dimensional Turbulence modeling. Proc. 11th Int. Symp. Turb. Shear Flow Phen. (TSFP11) (2019).

[26] H. Tanaka, S. Maruyama and S. Hatano. Combined forced and natural convection heat transfer for upward flow in a uniformly heated, vertical pipe. Int. J. Heat Mass Transfer (1987) 30(1):165-174. 